\title{
Numerical Simulation of Condensation of Natural Fog Aerosol under Acoustic Wave Action
}

\section{Aerosol and Air Quality Research}

\section{OPEN ACCESS}

Received: June 30, 2020

Revised: September 14, 2020

Accepted: September 22, 2020

${ }^{*}$ Corresponding Author:
zhouhefeng@nudt.edu.cn

\section{Publisher:}

Taiwan Association for Aerosol Research

ISSN: $1680-8584$ print

ISSN: 2071-1409 online

(c) Copyright: The Author(s). This is an open access article distributed under the terms of the Creative Commons Attribution License (CC BY 4.0), which permits unrestricted use, distribution, and reproduction in any medium, provided the original author and source are cited.

\author{
Chang Liu, Yun Zhao, Zhangfu Tian, Hefeng Zhou* \\ College of Meteorology and Oceanography, National University of Defence Technology, \\ Changsha, China
}

\section{ABSTRACT}

Acoustic condensation has proved its excellent performance in the dissipation of suspended fine particles. It is also promising for artificial fog dissipation, but the current research is still lacking. This paper sums up the mathematical model of acoustic condensation. Considering the physical properties of droplets, the improved root mean square (IRMS) acoustic condensation kernel function is proposed, which is compared with the analytic solution to verify the correctness. Through the method of numerical simulation, different condensation effects of the different condensation mechanisms are compared. Study the effect of acoustic frequency, sound pressure level and initial concentration on the condensation of natural fog aerosol under the action of sound waves. The results show that the droplet condensation efficiency can be improved by acoustic effect significantly; The effect of acoustic frequency on droplet condensation is nonlinear, and there is an optimal frequency of $600 \mathrm{~Hz}$. Besides, high-frequency sound wave has a better effect on small particles' condensation, while low-frequency sound wave has a better effect on large particles' condensation. The sound pressure level and initial particle concentration is positively correlated with the condensation efficiency, and there is a critical particle size. After the acoustic condensation, the concentration of particles larger than the critical particle size increases, and decreases conversely.

Keywords: Acoustic condensation, Natural fog, IRMS kernel function, Sectional method, Numerical simulation

\section{INTRODUCTION}

Fog weather has caused a great negative impact on people's daily life, it not only does harm to people's health, but also makes visibility seriously reduced, result in great safety risks on people's traffic travel. Traditional artificial fog dissipation can be divided into two specific operations: artificial warm fog dissipation and artificial cool fog dissipation (Gao et al., 2010). However, the traditional fog dissipation method is highly energy-consuming and corrosive, which is easy to cause secondary pollution, does more harm than good. Acoustic wave fog dissipation refers to use acoustic wave to radiate a fog field, so that droplets contact and collide with each other, condense into larger particles, achieve the effect of fog dissipation. Compared with the traditional fog dissipation method, Acoustic wave fog dissipation is a more promising technology.

In 1931, Patterson and Cawood (1931) first discovered acoustic agglomeration in the laboratory. In 1936, the Faraday Society in London held a meeting to study the feasibility of using sound waves for fog removal. During World War II, acoustic agglomeration technology was used in airports-defogging (Chou, 1980) to improve visibility over runways and airports. In 1963, Zhang et al. (1963) conducted a preliminary experimental study on the dispersing of water mist by sound waves. 'Rain gushes after lightning' is known as a sound-induced condensation acceleration phenomenon in nature. In 1964, Moore et al. (1964) recorded, measured, discussed and analyzed this phenomenon, and concluded that sound waves played an auxiliary role in promoting cloud condensation, while the discharge phenomenon of lightning promoted the mutual attraction between charged droplets, which was the main cause of this phenomenon. In 1965, Mednikov 
(1965) published the first book on acoustic agglomeration, summarized the previous research work of scholars in various countries, firstly established a systematic theory to explain the phenomenon of acoustic agglomeration, put forward the famous agglomeration mechanism (Orthokinetic Interaction Mechanism). The concept of agglomeration volume was introduced which refered to the area around a large particle that can agglomerate with a small particle, if the small particles appear in the agglomeration volume of a large particle, the collision and agglomeration can occur due to the difference of the entrainment coefficient between the two particles and the velocity difference. In 1979, Shaw and Tu (1979) adopted two acoustic sources, a low-frequency electro-acoustic horn (1-3 kHz) and a high-frequency horn (10-20 kHz), to conduct acoustic agglomeration experiments on monodisperse aerosols, and found that the agglomeration effect was better at low frequencies, while the acoustic attenuation was more serious at high frequencies. Rajendran et al. (1979) compared the agglomeration effect of aerosol under the flow and static conditions, and found that the agglomeration effect was reduced under the flow condition. He believed that the reason was the turbulence generated by the sound wave was suppressed by the airflow, so that the agglomeration caused by turbulence was reduced. In 1995, Caperan et al. (1995) studied the entrainment effect of ultrasonic field on ethylene glycol aerosol. The results show that sound condensation is affected by the amplitude of sound wave and is proportional to the quadratic square of the amplitude of sound wave.

Into the 21st century, Acoustic wave condensation technology has been widely used in suspension particle elimination (Hoffmann, 2000; Liu et al., 2009; Fan et al., 2013). In 2002, Hou et al. (2002) investigated the dissipation effect of low-frequency sound wave $(<50 \mathrm{~Hz})$ on water fog, and qualitatively concluded that lower frequency and higher sound intensity are conducive to the dissipation of water fog. In 2013, Peng (2013) carried out a study on the mechanism of oil mist condensation by ultrasonic wave through numerical simulation. In the simulation results, the condensation effect of particles becomes better with the increase of temperature, and worse with the increase of pressure. The increase of ultrasonic frequency is not conducive to the condensation of particles. The coagulation effect is the best when the ultrasonic frequency is at $20 \mathrm{kHz}$. As the coagulation effect decreases monotonously with the increase of the acoustic frequency in the simulation process, $20 \mathrm{kHz}$ is not the optimal frequency, and whether the ultrasonic wave is a favorable frequency band for acoustic coagulation also remains to be considered. Sahinoglu et al. (2013) studied the condensation effect of ultrasonic condensation on bubbles in liquid, and proposed a visualization method to describe the molecular trajectory of the aggregation process. In 2015, Tamara and Svetlana (2015) applied the acoustic condensation method to clouds and discussed the theoretical basis and feasibility of acoustic method to enhance rainfall. In 2018, Yu (2018) carried on the simulation and experiment of the ultrasonic condensation process of water mist, obtained that standing wave sound field is more likely to cause hydromechanical condensation between particles

At present, there are a lot of researches on the emission reduction of fine particle, the theoretical and experimental research on condensation of water mist (droplet) also has a certain basis, However, there are few studies on acoustic wave condensation for fog aerosol, especially under natural conditions. Fog drops have larger particle size, wider distribution spectrum, and different physical and chemical properties. At present, the known condensation mechanisms include orthokinetic interaction mechanism, acoustic wake mechanism, mutual radiation mechanism, gravity sedimentation mechanism and Brownian motion mechanism. Among them, the orthokinetic interaction mechanism is the most important condensation mechanism (Song, 1990; Liu, 2009). In the existing literature, most of the simulation of condensation process only uses one or two mechanisms for model establishment (Hoffmann and Koopmann, 1996; Hoffmann, 1997; Gonzalez et al., 2000; Cleckler et al., 2012), but in the actual condensation process, five mechanisms exist simultaneously, interact with each other and depend on each other (Wu, 2014).

In order to make the acoustic condensation technology economically applied in the field of defogging, it is necessary to study the influence of each parameter in the acoustic condensation process on the condensation effect, find out the "optimal operating parameters", make it work under the best conditions and reduce energy consumption, so that the acoustic condensation technology may become a practical technology. In the existing literature, the frequency range of acoustic waves adopted by researchers is very large, ranging from as low as $20 \mathrm{~Hz}$ to as high as $30 \mathrm{kHz}$ (Zhang et al., 1963; Hou et al., 2002; Yu, 2018). In these studies, various acoustic frequencies 
all produce a certain condensation effect, so the study of the optimal frequency is still controversial. In this paper, the present condensation kernel functions is fused and improved, a more practical mathematical model is proposed, and each condensation influence parameter is discussed, finally the optimal condensation parameters based on the natural fog condition are obtained.

\section{MODEL AND METHOD}

\subsection{Condensation Kernel Function}

The agglomeration process of fine particles can be simplified as Fig. 1, which shows the particle motion and condensation situation within a sound wave period in order from (a) to (e).

According to the above condensation mechanism, the number of collisions of suspended particles within a sound wave period in a condensed volume is:

$N_{i j}=\frac{1}{4} \pi\left(d_{i}+d_{j}\right)^{2} u_{i j} n_{i} n_{j}$

where, $d_{i}, d_{j}$ represents the particle sizes of particles with different particle sizes, $u_{i j}$ represents the average relative velocity of two particles with different particle sizes, $n_{i}, n_{j}$ represents the number of particles with different particle sizes.

The acoustic condensation kernel function is defined as the number of collisions between particles with different concentrations and particle sizes per unit time, which can be expressed as:

$K_{i j}=N_{i j} / n_{i} n_{j}$

By synthesizing Eq. (1) and (2), the condensed kernel function can be obtained:

$K_{i j}=\frac{1}{4} \pi\left(d_{i}+d_{j}\right)^{2} u_{i j}$

It can be seen from Eq. (3) that the key to solving the condensation kernel function lies in solving the relative velocity of two kinds of particle size particles in the sound field, so the motion state law of particles in the sound field needs to be solved. If the crushing and loss in the condensation process is ignored, the number of particles with volume $v$ changes with time, and it should be as follows:

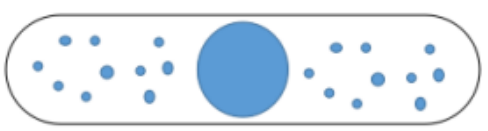

(a) $\mathrm{t}=0$

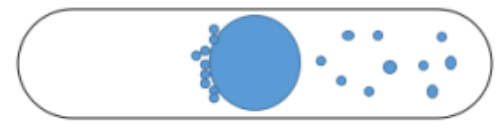

(c) $\mathrm{t}=0.5 \mathrm{~T}$

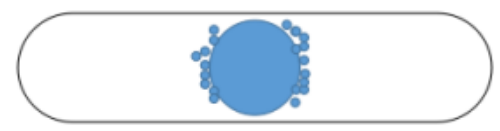

(e) $\mathrm{t}=\mathrm{T}$

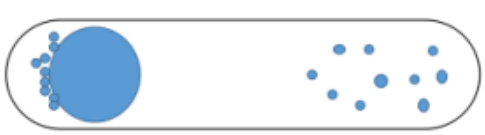

(b) $\mathrm{t}=0.25 \mathrm{~T}$

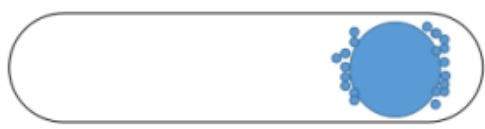

(d) $\mathrm{t}=0.75 \mathrm{~T}$

Fig. 1. Simplified particle condensation process. 


$$
\frac{\partial n(v, t)}{\partial t}=\frac{1}{2} \int_{0}^{v} K\left(v-v^{\prime}, v^{\prime}\right) n\left(v-v^{\prime}, t\right) n\left(v^{\prime}, t\right) d v^{\prime}-n(v, t) \int_{0}^{v} K\left(v, v^{\prime}\right) n\left(v^{\prime}, t\right) d v^{\prime}
$$

where, $n(v, t)$ is the particle number concentration.

Transform Eq. (4) into a discrete Smoluchowski equation as follows:

$$
\frac{d n_{k}}{d t}=\frac{1}{2} \sum_{i+j=k} K(i, j) n_{i} n_{j}-n_{k} \sum_{i=1}^{m} K(i, k) n_{i}
$$

By integrating Eq. (5) with the particle size, the relationship between the total number of discrete aerosol particles $\left(N_{t}\right)$ and time $(t)$ can be obtained:

$$
\frac{d N_{k}}{d t}=-\frac{1}{2} \sum_{i=1}^{m} \sum_{j=1}^{m} K(i, j) n_{i} n_{j}
$$

Here is based on the law of conservation of mass, assuming that after the two particles collide, forming new sphere or aggregate immediately. Eq. (6) shows the relationship between the change rate of suspended particle number and agglomeration kernel function. The acoustic agglomeration kernel function can be used to estimate the change of the number of suspended particles and qualitatively describe the acoustic agglomeration effect of suspended particles. Therefore, acoustic condensation kernel function is the most important key in research of acoustic condensation

\subsubsection{Orthokinetic interaction mechanism}

Due to the transmission of sound wave, the velocity of fluid medium changes periodically, particles are entrained by sound wave. The motion speed of suspended particles is different from that of surrounding media, particle vibration velocity behind the acoustic vibration velocity in the medium. Thus, in the sound field, different size particles collide with each other and condense into one may happen.

The force $F$ of the fluid medium on the particle can be expressed as:

$F=3 \pi \eta d\left(u_{g}-u_{p}\right)$

where, $u_{p}$ stands for particle velocity, $u_{g}$ stands for medium velocity. Ignoring the influence of gravity and other factors, in a gas medium with a low Reynolds number, according to Newton's second law, the force equation of particles with a mass of $m_{p}$ (Wang, 2012) is:

$F=m_{p} \frac{d u_{p}}{d t}$

Eq. (8) is an ordinary differential equation of first order. When the initial conditions are satisfied $t=0$ and the particle is at rest $u_{p}=0$, the particle motion expression can be solved as follows:

$u_{p}=\frac{u_{0} \sin (\omega t-\varphi)}{\sqrt{1+\left(\omega \tau_{d}\right)^{2}}}+\frac{\omega \tau_{d} u_{0} e^{-t / \tau_{d}}}{1+\left(\omega \tau_{d}\right)^{2}}$

where, the relaxation time of the particles is: $\tau_{d i}=\rho_{p} d_{i}^{2} / 18 \mu, \varphi$ refers to the vibration phase difference between particles and fluid medium, $\omega$ is the angular frequency, the former term of Eq. (9) is the steady-state solution, while the latter term is the transient solution. In general, the transient term will rapidly reduce to 0 , and the particle motion becomes the steady-state:

$u_{p}=\frac{u_{0} \sin (\omega t-\phi)}{\sqrt{1+\left(\omega \tau_{d}\right)^{2}}}$ 
The ratio of particle amplitude to fluid medium amplitude is defined as particle entrainment coefficient $\mu_{p}$.

$\mu_{p}=\frac{u_{p}}{u_{g}}=\frac{1}{\sqrt{1+\omega^{2} \tau_{d}^{2}}}=\cos \phi$

According to the entrainment coefficient, the motion equation of the particle is:

$u_{p}=u_{0} \mu_{p} \sin (\omega t-\varphi)$

It can be seen that the entrainment capacity for particles with the same particle size, the same sound pressure level and different frequencies is different, and the entrainment capacity for particles with different particle size, the same sound pressure level and the same frequency is also different.

From the Eq. (12), the average value of the relative velocity of particles with different particle sizes in a sound wave period can be obtained:

$u_{i j}=\frac{2}{\pi} \frac{u_{0} \omega\left|\tau_{d i}-\tau_{d j}\right|}{\sqrt{1+\left(\omega \tau_{d i}\right)^{2}} \sqrt{1+\left(\omega \tau_{d j}\right)^{2}}}$

The orthokinetic interaction condensation kernel function can be obtained as follows:

$K_{i j}^{O r}=\frac{1}{2} u_{0}\left(\mathrm{~d}_{\mathrm{i}}+\mathrm{d}_{\mathrm{j}}\right)^{2} \frac{\omega\left|\tau_{d i}-\tau_{d j}\right|}{\sqrt{1+\left(\omega \tau_{d i}\right)^{2}} \sqrt{1+\left(\omega \tau_{d j}\right)^{2}}}$

From Eq. (14), it can be seen that the orthokinetic interaction condensation of particles with the same particle size is 0 , so obviously the orthokinetic interaction condensation mechanism is not perfect.

\subsubsection{Mutual radiation pressure force mechanism}

When the particles are entrained to move in the sound field, the fluid velocity between the particles is larger than that outside the particles. According to Bernoulli equation, this difference in velocity will create a net pressure for the particles to attract each other.

If the mutual radiation pressure is stable, the particle velocity caused by fluid mechanics can be expressed as:

$u_{h}=F / 3 \pi \mu d$

The tangential velocity between particles is not taken into account, but the radial velocity between particles is taken into account, because the radial velocity is the cause of particle collision and has nothing to do with whether the particle is rotated or not. The velocity of particle $\mathrm{j}$ relative to particle $\mathrm{i}$ is:

$u_{i j}^{H y h}=\frac{1}{3 \pi \mu}\left(\frac{f_{j i}}{d_{j}}-\frac{f_{i j}}{d_{i}}\right)$

The radial component of $u_{i j}^{H y h}$ determines whether two particles collide, and the radial component of the relative velocity is called convergence velocity. The interaction hydrodynamic force of particle $j$ and particle $i$ is in the opposite direction, and the motion of particle $j$ towards particle $i$ is set as the positive direction, so the convergence velocity can be expressed as a scalar: 


$$
u_{i j}^{H y h}=\frac{1}{3 \pi \mu}\left(-\frac{f_{j i r}}{d_{j}}-\frac{f_{i j r}}{d_{i}}\right)=\frac{\rho_{0} u_{0}^{2} a_{i}^{2} a_{j}^{2}\left(1-3 \cos ^{2} \theta\right)}{16 \pi^{2} r^{4} \mu}\left(a_{i} g_{j i r}+a_{j} g_{i j r}\right)
$$

where $g_{i j}$ is a fluid mechanics interaction function. After numerical verification, $g_{i j}$ is always positive.

The kernel function of the mutual radiation condensation mechanism is obtained:

$$
K_{i j}^{H y h}=\frac{\sqrt{3} \rho_{0} u_{0}^{2} a_{i}^{2} a_{j}^{2}\left(a_{i}+a_{j}\right)^{2}}{18 \pi \mu r^{4}} g_{j i}
$$

\subsubsection{Acoustic wake effect mechanism}

The acoustic wake effect is based on Oseen (Re < 10) (Pijush and Ira, 2008), the asymmetry of the flow field around the particles. When there is a relative velocity between the particles and the fluid, the particles disturb the flow field on both sides of the front and the back differently, and the disturbance on the back is greater, forming a low-pressure wake. The motion trajectory of aerosol particles in the sound field captured by microscopic high-speed photography has verified the existence of acoustic wake effect, which is considered to be one of the main condensation mechanisms of monodisperse aerosol (Hoffmann and Koopmann, 1994).

One of the assumptions of acoustic wake effect is that the particle size is much smaller than the acoustic boundary layer thickness. As can be seen from the definition, there is a frequency limit, high frequency does not apply, low frequency applies, so the hypothesis of low frequency should be attached to the mechanism of acoustic wake effect condensation, otherwise it will not be valid.

The force on the particle in the Oseen flow should be modified:

$F=3 \pi \mu d\left(u_{g}-u_{p}\right)\left(1+\frac{3}{8} \operatorname{Re}\right)$

By combining Eq. (8), the particle force equation (Hoffmann and Koopmann, 1994) in Oseen flow can be obtained as follows:

$\frac{d u_{p}}{d t}+\frac{u_{p}}{\tau_{d}}=\frac{u_{g}}{\tau_{d}}+\frac{3}{16} \frac{d}{v \tau_{d}}\left(u_{g}-u_{p}\right)^{2}$

After derivation, the convergence speed is:

$$
\begin{aligned}
& u_{i j}^{H y s}=\frac{3 u_{0}}{8 \pi r_{0}}\left[d_{i} I_{i}+d_{j} I_{j}+\frac{U_{0}}{2 \pi v}\left(d_{i}^{2} I_{i}^{2}+d_{j}^{2} l_{j}^{2}\right)\right]-\frac{3 v}{\pi^{2} r_{0}^{2}}\left(d_{i}+d_{j}\right) \\
& -\frac{9 U_{0}}{64 \pi r_{0}^{2}}\left(d_{i}^{2} l_{i}+d_{j}^{2} l_{j}\right)+\frac{3 U_{0}^{2}}{16 \omega r_{0}^{2}} l_{i} l_{j}\left(l_{i} q_{i}-l_{j} q_{i}\right)\left(d_{i}-d_{j}\right)
\end{aligned}
$$

where, $I$ is the slip coefficient of the Oseen flowing region, $q$ is the entrainment coefficient of particles in the Oseen flowing region, $r_{0}$ is the particle spacing, and the dynamic viscosity constant is $v=\mu / \rho_{g}$.

When the particle sizes are the same:

$u_{i j}^{H y s}=\frac{3 u_{0}}{8 \pi r_{0}}\left(2 d_{i} I_{i}+\frac{U_{0}}{\pi v} d_{i}^{2} I_{i}^{2}\right)-\frac{6 v}{\pi^{2} r_{0}^{2}} d_{i}-\frac{9 U_{0}}{32 \pi r_{0}^{2}} d_{i}^{2} I_{i}$

The kernel function of the acoustic wake effect condensation mechanism is obtained: 


$$
\begin{aligned}
& K_{i j}^{\text {Hys }}=\frac{1}{4} \pi\left(d_{i}+d_{j}\right)^{2} \mid \frac{3 u_{0}}{8 \pi r_{0}}\left[d_{i} I_{i}+d_{j} I_{j}+\frac{U_{0}}{2 \pi v}\left(d_{i}^{2} l_{i}^{2}+d_{j}^{2} I_{j}^{2}\right)\right]-\frac{3 v}{\pi^{2} r_{0}^{2}}\left(d_{i}+d_{j}\right) \\
& -\frac{9 U_{0}}{64 \pi r_{0}^{2}}\left(d_{i}^{2} I_{i}+d_{j}^{2} I_{j}\right)+\frac{3 U_{0}^{2}}{16 \omega r_{0}^{2}} I_{i} I_{j}\left(I_{i} q_{i}-I_{j} q_{i}\right)\left(d_{i}-d_{j}\right) \mid
\end{aligned}
$$

\subsubsection{Gravity sedimentation mechanism}

The gravity sedimentation velocity of particles with the same density and different particle sizes or the same particle size and different density is different, so that the particles will have relative motion under the action of gravity, which can promote the collision and condensation between the suspended particles.

For suspended fine particles, the resistance of spherical particles settling in viscous fluid in an infinite range can be assumed to be completely caused by the viscosity of the fluid, so the viscous resistance can be expressed as Eq. (7).

Due to the discontinuity of air molecules, the viscous resistance in the air medium should be corrected.

$F_{d r a g}=3 \pi d_{i} \mu_{G} / C$

where, $C$ stands for Cunningham slip correction coefficient (Wang, 2007), $u_{G}$ is the sedimentation velocity.

According to Newton's second Law, $v$ is the particle volume, $g$ is the gravity constant, and the force on the particle in the direction of gravity can be expressed as (John and Peter, 2006):

$\rho_{p} \mathrm{vg}=\rho_{g} \mathrm{vg}+F_{d r a g}$

where, $\rho_{p}$ is single particle density, $\rho_{g}$ air density.

Then it can be concluded that the relative velocity caused by gravity sedimentation is:

$u_{i j}^{G r}=\frac{\left(\rho_{P}-\rho_{g}\right) g\left(d_{i}^{2}-d_{j}^{2}\right)}{18 \mu}$

By substituting into Eq. (3), the condensation kernel function of gravity settlement is as follows:

$K_{i j}^{G r}=\frac{\pi \rho_{p} g\left(d_{i}+d_{j}\right)^{2}\left(d_{i}^{2}-d_{j}^{2}\right)}{72 \mu}$

\subsubsection{Brownian motion mechanism}

The particles collide with each other and condense because of their ceaseless, irregular Brownian motion. Brown condensation plays an important role in the agglomeration of subfine particles. The average particle size of fog drops is around $5 \pi \mathrm{m}$, so Brown motion can not be ignored (Wang, 2012).

Brown condensation occurs between two particles with radius $a_{i}$ and $a_{j}$ in the sound field. When the aggregation time is long enough $t \rightarrow \infty$, let $n_{j}$ be the concentration of particle $\mathrm{j}$ and the number of particle collisions in the stable state is:

$N_{i j}=\lim _{t \rightarrow \infty} N_{f}(t)=4 \pi D\left(a_{i}+a_{j}\right) n_{i} n_{j}$

According to Einstein equation, the diffusion coefficient D can be solved as follows:

$D_{i j}=D_{i}+D_{j}$

Substitute Eq. (29) into Eq. (28). 
$N_{i j}=4 \pi\left(D_{i}+D_{j}\right)\left(a_{i}+a_{j}\right) n_{i} n_{j}$

The kernel function of Brownian condensation mechanism can be obtained as follows:

$K_{i j}^{B r}=\frac{N_{i j}}{n_{i} n_{j}}=4 \pi\left(D_{i}+D_{j}\right)\left(a_{i}+a_{j}\right)$

\subsection{Numerical Computation Method}

At present, the Population Balance Model (PBM) (Yao et al., 2015) is usually used to describe the distribution change of particle size in the gas-liquid system and solve it through the Population Balance Equation (PBE), namely Eqs. (4), (5), and (6).

Through the calculation of condensation kernel function can estimate two kinds of particles' collision situation and number change. Then the particle size distribution change of a particle group needs to find a method to solve the PBE. There are three mainstream methods to solve the PBE, namely, sectional method (Ezekoye and Wibowo, 1999; Zhang et al., 2012), moment method (Zhang et al., 2011) and Monte Carlo method (Tandon and Rosner, 1999; Sheng and Shen, 2006; Sheng and Shen, 2007). Considering the calculation amount and calculation accuracy, this paper chooses the sectional method to solve the particle group balance equation.

The sectional method discretizes the particle size distribution curve (Zhang, 2010). The balance equations of the global parameters of a particle scale spectrum are established for each discrete interval, and then the common difference equations are solved simultaneously.

The maximum and minimum particle sizes in aerosol are respectively $d_{u p}$ and $d_{\text {low. }}$ In any region, the maximum particle size is $d_{i, \max }$, the minimum particle size is $d_{i, \min }$, average particle size is $d_{i, a v e .}$ When the particles in region $\mathrm{i}$ and $\mathrm{j}$ condense, the particle size range produced is $d_{i, \min }+d_{j, \min }-$ $d_{i, \max }+d_{j, \max }$, when the particle size range includes part or all of this range, the condensed particles may fall into it. However, the probability of particles falling into each region after condensation is not the same. Assuming that $d_{i, a v e}<d_{j, \text { ave }}$, the following relation must exist:

$d_{i, \min }<d_{i, \max } \leq d_{j, \min }<d_{j, \max }$

$d_{i, \max }-d_{i, \min } \leq d_{j, \max }-d_{j, \min }$

As shown in Fig. 2, the cartesian coordinate system was established with particle size as the coordinate axis, and the probability of particle size falling into each region after condensation was calculated. Where, rectangle $A B C D$ represents the region where particle size may fall after condensation, and the particle sizes corresponding to its four vertices are respectively: $A \rightarrow d_{i, \min }$ $+d_{j, \min } ; B \rightarrow d_{i, \min }+d_{j, \min } ; C \rightarrow d_{j, \max }+d_{i, \min } ; D \rightarrow d_{i, \max }+d_{j, \max }$. The line segment EF forms a $45^{\circ}$
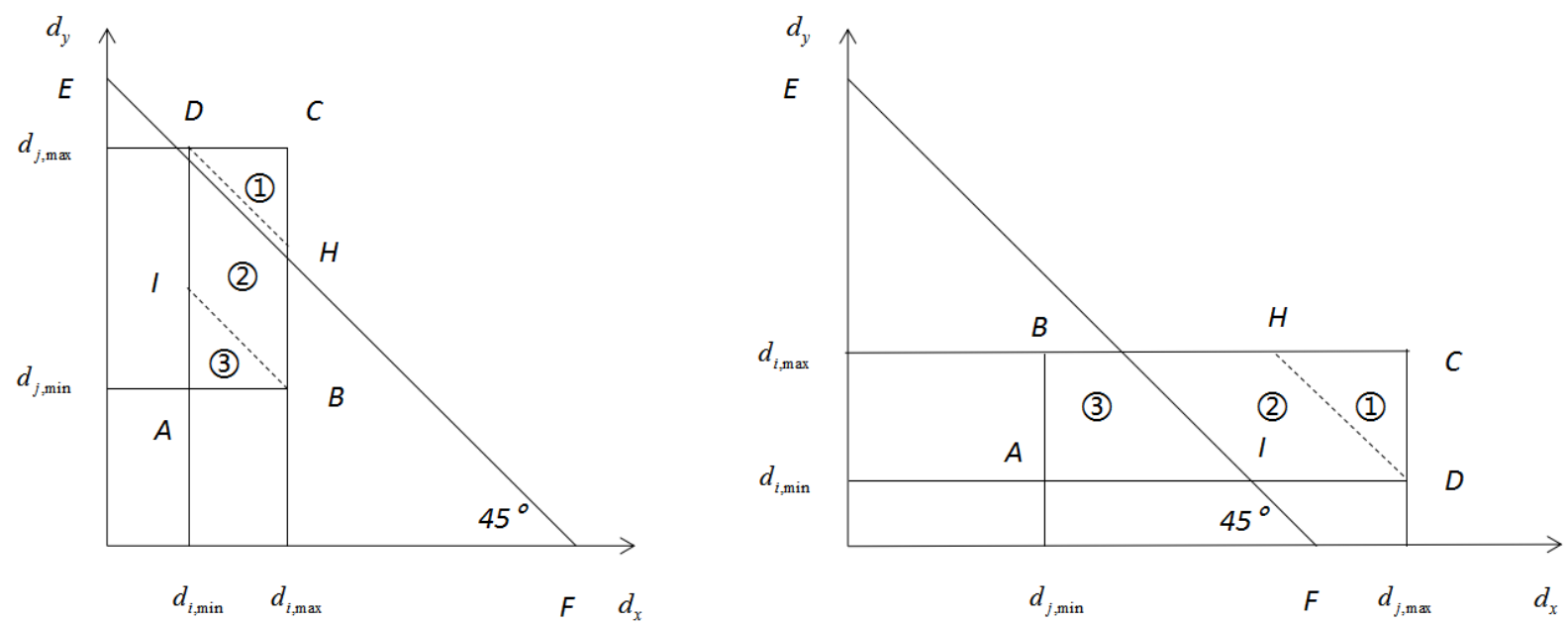

Fig. 2. Probability calculation of particle size distribution after condensation. 
angle with both $d_{i}$ and $d_{j}$ axis, its expression is $d_{x}+d_{y}=d_{c}$ ( $d_{c}$ is an arbitrary constant, whose physical meaning is: the particle size of particles falling on the line segment EF is the same). The translation process of the line segment represents the change of particle size after condensation, its intersection with the region $\mathrm{CDH}, \mathrm{DIBH}, \mathrm{ABI}$ indicates that the particle size will fall into the corresponding region after condensation. Express the corresponding particle size in different regions as a set, the set of particle sizes corresponding to $\mathrm{CDH}$ in the region is: (1) $=\left\{d_{c} \mid d_{i, \min }+\right.$ $d_{j, \min }<d_{c}<d_{i, \max }+d_{j, \min \}}$; the set of particle sizes corresponding to $\mathrm{DIBH}$ in the region is: (2) = $\left\{d_{c} \mid d_{i, \max }+d_{j, \min }<d_{c}<d_{j, \max }+d_{i, \min }\right\}$; the set of particle sizes corresponding to $A B I$ in the region is: (3) $=\left\{d_{c} \mid d_{j, \text { max }}+d_{i, \text { min }}<d_{c}<d_{j, \text { max }}+d_{i, \text { max }}\right\}$. The difference between the two figures in Fig. 2 is that the particle size in region $\mathrm{i}$ and $\mathrm{j}$ is expressed on different axes, the probability of particle size $d_{a}$ $<d_{c}$ after condensation is the same.

Assume that the particle size generated after condensation is $d_{a}$, the probability of $d_{a}<d_{c}$ is $P\left(d_{a}<d_{c}\right)$, and its value is obtained by dividing the area of rectangle ABCD at the lower left of the line segment EF by the total area of rectangle $A B C D$.

$$
P\left(d_{a}<d_{c}\right)=\left\{\begin{array}{l}
0, d_{c}<d_{i, \text { min }}+d_{j, \text { min }} \\
\frac{\left(d_{c}-d_{i, \text { min }}-d_{j, \text { max }}\right)^{2}}{2\left(d_{j, \text { max }}-d_{j, \text { min }}\right)\left(d_{i, \text { max }}-d_{i, \text { min }}\right)}, d_{c} \in(1) \\
\frac{0.5\left(d_{i, \text { max }}-d_{i, \text { min }}\right)+\left(d_{c}-d_{j, \text { min }}-d_{i, \text { max }}\right)}{\left(d_{j, \text { max }}-d_{j, \min }\right)}, d_{c} \in(2) \\
1-\frac{\left(d_{i, \text { max }}+d_{j, \text { max }}-d_{c}\right)^{2}}{2\left(d_{j, \text { max }}-d_{j, \text { min }}\right)\left(d_{i, \text { max }}-d_{i, \text { min }}\right)}, d_{c} \in(3) \\
1, d_{c}<d_{i, \text { min }}+d_{j, \text { min }}
\end{array}\right.
$$

Then, according to $\mathrm{P}$, the condensed particles were regrouped and solved iteratively until the number of particles reached the set threshold. The iteration ended and the condensation process simulation was completed.

\subsection{Improvement of Mathematical Model}

The key of acoustic condensation simulation is to determine the condensation kernel function, Among all kinds of existing condensation mechanisms, the most important one is the orthokinetic interaction condensation mechanism, its universality is the highest, however, it also has a disadvantage that the monodisperse particles can not be simulated. In the acoustic condensation process of particles, various condensation mechanisms are coordinated and partially overlapped with each other, their influence on the process of the collision and condensation of different size particles is different, so need to find a more comprehensive and reasonable kernel function.

In 1977, Volk (1977) first carried out numerical simulation of acoustic wave condensation, which was mainly based on the orthokinetic interaction condensation mechanism, supplemented by secondary effects such as acoustic radiation force, and added up all condensation kernel functions. The simulation results were lower than the experimental values; In 1980, Chou (1980) adopted the orthokinetic interaction mechanism and turbulence condensation mechanism (it was generated when the sound pressure level was greater than $160 \mathrm{~dB}$, which was difficult to achieve in reality and harmful to human body, which was not considered in this paper), and the simulation results could not reasonably describe the acoustic condensation process; In 1981, Patel (1981) adopted the orthokinetic interaction mechanism and the mutual radiation mechanism, the particle size distribution at each moment needed to be constructed as a normal distribution. In the same year, Cheng (1981) only considered the orthokinetic interaction mechanism and Brownian motion mechanism, the model was still relatively rough; In 1990, Song (1990) adopted the orthokinetic interaction mechanism, mutual radiation mechanism and mutual scattering mechanism, the effect has improved; In 1994, Temkin (1994) only adopted the orthokinetic interaction mechanism and made a lot of simplification, the model could only simulate the condensation situation within a narrow range of particle sizes; In 2006, Sheng and 
Shen (2006) added Brownian motion mechanism, orthokinetic interaction mechanism, fluid mechanics mechanism (including mutual radiation mechanism, acoustic wake effect mechanism) and gravity sedimentation mechanism into the model, which was found to be in good agreement with the experimental results; In 2019, Zheng and Wang (2019) use orthokinetic interaction mechanism, fluid mechanics mechanism and Brownian motion mechanism, put forward a new mode of coupling that the root mean square condensation kernel function, simulation effect was better, and found that the new coupling mode was more consistent with the fact that the condensation effect of particles in the submicron region decreased, and the fact that the condensation effect decreased when the particle sizes of two particles involved in collision condensation were sufficiently close.

Synthesize the previous research results. fog drops' average particle size is larger, the distribution spectrum is wider, the concentration is relatively small, and the condensation time is relatively long, the condensation kernal function of gravity sedimentation is positively correlated with the particle size ratio of suspended particles. The larger the particle size ratio is, the larger the kernel function of gravity sedimentation is, the more favorable it is for the occurrence of gravity sedimentation (John and Peter, 2006). Therefore, on the basis of the orthokinetic interaction mechanism and the hydromechanics condensation mechanism, the gravity sedimentation mechanism is added. Fluid mechanics condensation mechanism can make up for the theoretical defect of orthokinetic interaction condensation and gravity sedimentation mechanism that monodisperse droplets particles cannot be condensed. Among hydromechanics condensation mechanisms, acoustic wake mechanism is the main fluid mechanics mechanism of condensation (Hoffmann, 1997; González et al., 2001), explains the condensation principle when sound wave direction is parallel to the two particles, while, mutual radiation mechanism explains the principle of condensation when the sound wave direction is verticle to the two particles (González et al., 2002, 2003), so this article adds the mutual radiation condensation mechanism so that the hydromechanics condensation mechanism can be more perfect. In the last paragraph, the simulation effect added Brownian motion mechanism is generally better, thus the importance of Brownian motion mechanism was confirmed. Finally, the improved root-meansquare (IRMS) condensation kernel function is obtained by coupling the required mechanisms through the root-mean-square coupling method. the expression is:

$$
K_{i j}=\sqrt{K_{i j}^{O r 2}+K_{i j}^{H y s 2}+K_{i j}^{H y h 2}+K_{i j}^{G r 2}+K_{i j}^{B r 2}}
$$

\subsection{Particle Size Distribution of Fog}

In the numerical simulation of fog aerosol, the initial particle size distribution is a very important parameter. Referring to Li and Niu's (2001) summary of Chinese fog in the middle and late 20th century, according to the different locations of fog, there are mainly urban fog, mountain fog and sea fog. The number concentration of urban fog is $10^{2}-10^{3} \mathrm{~cm}^{-3}$, and the average diameter is less than $10 \mu \mathrm{m}$. The number concentration of sea fog is $10-10^{2} \mathrm{~cm}^{-3}$, and the average diameter is $22.1 \mu \mathrm{m}$. The number concentration of mountain fog is $10^{2} \mathrm{~cm}^{-3}$, and the average diameter is between city fog and sea fog. Inland fog (urban fog and mountain fog). According to how they formed, fog can be divided into advection radiation fog, advection fog, radiation fog and rain fog. In this paper, the observation data of fog drop spectrum in Nanjing of Liu (Niu and Lu, 2016) are referred to as experimental reference data. The instrument used is the FM-100 fog drop spectrometer of DMT Company of The United States, see Table 1. for parameters.

There are mainly three distribution models of natural fog, namely, exponential spectrum distribution, power spectrum distribution and lognormal distribution (Li and Guan, 2009). The lognormal distribution is the most commonly used spectral distribution at present, and its function expression is:

$$
n(r)=\frac{d N}{d r}=\frac{N_{0}}{\sqrt{2 \pi} \cdot \ln 10 \cdot r \cdot \ln \sigma_{g m}} \cdot \exp \left[-\frac{\left(\ln r-\ln r_{g m}\right)^{2}}{2 \ln ^{2} \sigma_{g m}}\right]
$$

where, $N_{0}$ is the total number of particles in the air per unit volume; $r_{g m}$ and $\sigma_{g m}$ are two 
Table 1. Observed data of Nanjing fog droplet spectrum.

\begin{tabular}{lllll}
\hline & $\begin{array}{l}\text { Droplet number } \\
\text { concentration }\left(\mathrm{cm}^{-3}\right)\end{array}$ & $\begin{array}{l}\text { Water content } \\
\left(\mathrm{g} \mathrm{m}^{-3}\right)\end{array}$ & $\begin{array}{l}\text { Average diameter } \\
(\mu \mathrm{m})\end{array}$ & $\begin{array}{l}\text { Spectral width } \\
(\mu \mathrm{m})\end{array}$ \\
\hline Advection radiation fog & 211 & 0.12 & 5 & $\geq 45$ \\
Advection fog & 108 & 0.021 & 3.6 & $\geq 45$ \\
Radiation fog & 78 & 0.023 & 3.7 & $\geq 35$ or $\leq 15$ \\
Rain fog & 3 & $0.00004-0.00021$ & 3.1 & $\leq 7$ \\
\hline
\end{tabular}

characteristic parameters describing the lognormal distribution, respectively called geometric mean radius and geometric standard deviation. In principle, the logarithmic normal distribution (including their superposition) can basically describe the entire aerosol scale spectrum distribution as long as appropriate parameters are selected.

To sum up, advective fog is the most common in urban fog, and its parameters are moderate and representative. Therefore, the particle size distribution of fog drops in this paper adopts the logarithmic normal distribution of advective fog, the average diameter of fog drops was $3.5 \mu \mathrm{m}$, the minimum particle size was $0.2 \mu \mathrm{m}$, the maximum diameter was $45 \mu \mathrm{m}$, the initial concentration was $100 \mathrm{~cm}^{-3}$, as shown in Fig. 3 .

\section{RESULTS AND DISCUSSION}

\subsection{Correctness Verification of Acoustic Condensation Model}

There is generally no analytical solution to the PBE due to the complex condensation kernel function caused by the actual physical and chemical processes. However, in some ideal cases, when the kernel function is very simple, there are analytic solutions to the PBE.

According to the research (Scott, 1968), for the aerosol with exponential initial particle size distribution, when the condensation kernel function meets one of the following three conditions, there is an analytical solution for simple condensed aerosol dynamic events:

(1) The condensation kernel is constant

$K_{i j}=a$

(2) The condensation kernel function is proportional to the sum of the volumes of the two particles

$K_{i j}=b\left(v_{i}+v_{j}\right)$

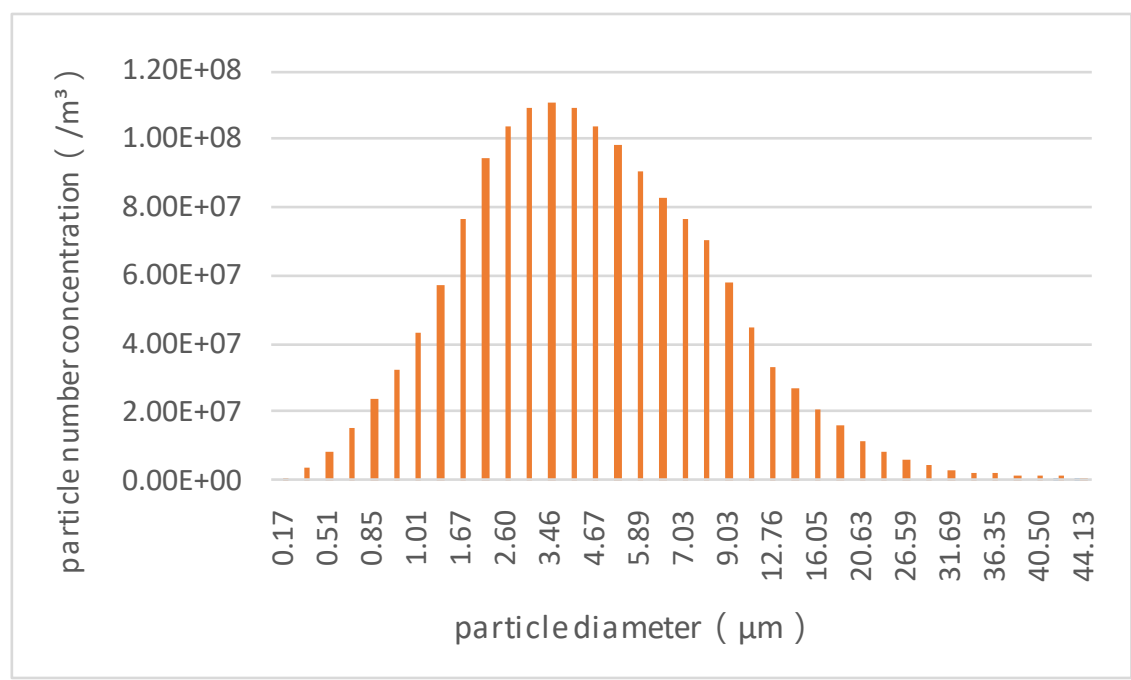

Fig. 3. Lognormal particle size distribution of advection fog. 
(3) The condensation kernel function is proportional to the volume product of the two particles

$K_{i j}=c v_{i} v_{j}$

where $a, b$ and $c$ are constants.

In this paper, we chose the simplest condition of condensation kernel function was constant, and compared the analytical solution with the numerical results to verify the correctness of the numerical model algorithm.

The initial particle size distribution of the aerosol with exponential distribution can be calculated as follows:

$n(v)=\frac{N_{0}}{v_{0}} \exp \left(-\frac{v}{v_{0}}\right)$

where, $N_{0}$ is the total aerosol particle number concentration, $v_{0}$ is the average volume of aerosol particles, and when the condensation kernel function is $k_{0}$, the relationship between aerosol particle size distribution and condensation time (Gelbard and Seinfeld, 1978) is:

$n(v, t)=\frac{4 N_{0}}{v_{0}(\tau+2)^{2}} \exp \left(-\frac{2 v / v_{0}}{\tau+2}\right)$

$\tau=N_{0} k_{0} t$

The selected calculation conditions are as follows: the temperature is $20^{\circ} \mathrm{C}$; The constant condensation kernel function is $k_{0}=5 \times 10^{-7} \mathrm{~s}^{-1}$; The average particle size of fog drops is $1.5 \mu \mathrm{m}$, the maximum particle size is $3 \mu \mathrm{m}$, and the minimum particle size is $0.2 \mu \mathrm{m}$. The initial particle number concentration is $1 \times 10^{4} \mathrm{~m}^{-3}$; Collision efficiency is 1 ; The number of groups is 50 .

Fig. 4. compares the aerosol particle size distribution obtained by numerical simulation with that obtained by analytical solution. The continuous solid line in the figure is the calculation result of numerical simulation, and the discrete points are the calculation result of analytical solution. It can be seen from the figure that the numerical simulation results are basically in agreement with the analytical solution calculation results, while in the range of particle sizes smaller than $1 \mu \mathrm{m}$, the coincidence degree is relatively poor. This is because the analytical solution simplifies the condensed kernel function approximately, the results obtained will not be identical to those obtained by numerical simulation. In numerical simulation the IRMS

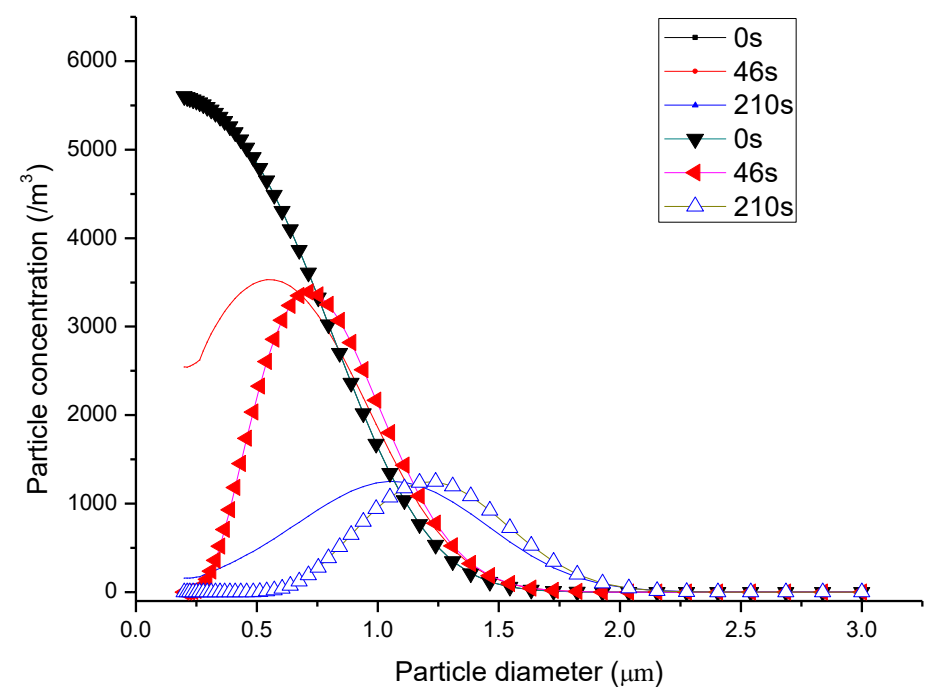

Fig. 4. Comparison between condensation of numerical simulation and analytical solution. 
condensation kernel function takes into account the situation that the particle collision efficiency decreases in the interval of submicron particles, so the particle concentration is higher than those obtained by analytical solution after acoustic wave action.

On the whole, the numerical simulation results of aerosol subjected to acoustic wave for different time are similar to the analytical solution results. In the range of particle sizes larger than $1 \mu \mathrm{m}$, the simulation value is basically consistent with the analytical value. Therefore, it can be concluded that the improved acoustic condensation model algorithm can effectively simulate the condensation process of natural fog and verify the correctness of the model.

\subsection{Condensation Simulation of Natural Fog under Acoustic Wave Action}

Parameter conditions: the temperature was $20^{\circ} \mathrm{C}$, the average diameter of fog drops was $3.5 \mu \mathrm{m}$, the minimum particle size was $0.2 \mu \mathrm{m}$, the maximum diameter was $45 \mu \mathrm{m}$, the initial concentration was $100 \mathrm{~cm}^{-3}$, the collision efficiency was 1 , the number of groups was 50 , the frequency was $600 \mathrm{~Hz}$, and the sound pressure level was $160 \mathrm{~dB}$.

Fig. 5(a) shows the particle size distribution curve of the condensation of fog aerosol at different times by using the IRMS condensation mechanism. It can be seen that under the given parameters, the sound waves had the condensation effect on fog drops, and it needed $1280 \mathrm{~s}$ to achieve the condensation effect of $90 \%$. As can be seen from Fig. 6, the effect of sound wave plays a very important role in the condensation and dissipation of fog drops. Similarly, after $1200 \mathrm{~s}$, the condensation efficiency with sound wave effect is more than twice as high as that with silent wave effect.

Fig. 6 shows the condensation effect of different mechanisms under the condition of natural fog. The control action time was $1200 \mathrm{~s}$, and the condensation effects of fog drops were respectively compared with the non-acoustic wave, the traditional orthokinetic interaction mechanism, the fluid mechanics action mechanism, the IRMS condensation mechanism and the linear superposition condensation mechanism. First of all, when there is no external acoustic wave, the condensation of fog drops is all gravitational sedimentation, and the condensation effect is weak. By comparing the IRMS condensation mechanism, it shows that the effect of acoustic wave on the condensation efficiency of fog drops is significant. The graph of orthokinetic interaction mechanism and the IRMS mechanism and linear superposition mechanism are similar, and the condensation effect is prominent. Overall, condensation efficiency: linear superposition $>$ IRMS > orthokinetic interaction. This is because the orthokinetic interaction mechanism is defective as a single condensation mechanism and cannot reflect all phenomenon of actual condensation, so the condensation effect is poor. The IRMS condensation mechanism is weaker than the linear superimposed condensation mechanism, which also confirms again that the IRMS condensation mechanism considers that the worse condensation effect between submicron particles or particle sizes are the same, it's closer to reality; The hydrodynamic mechanism, as

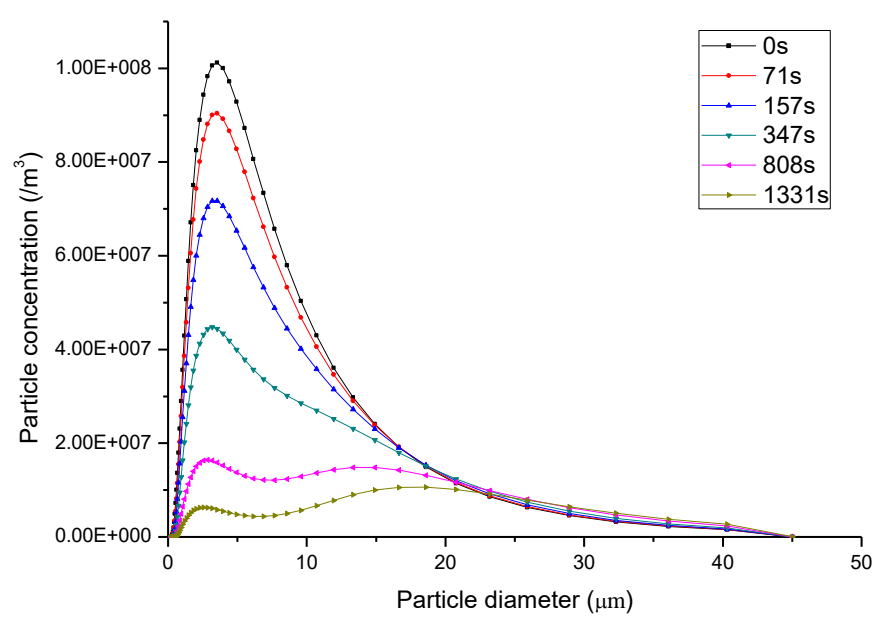

(a)

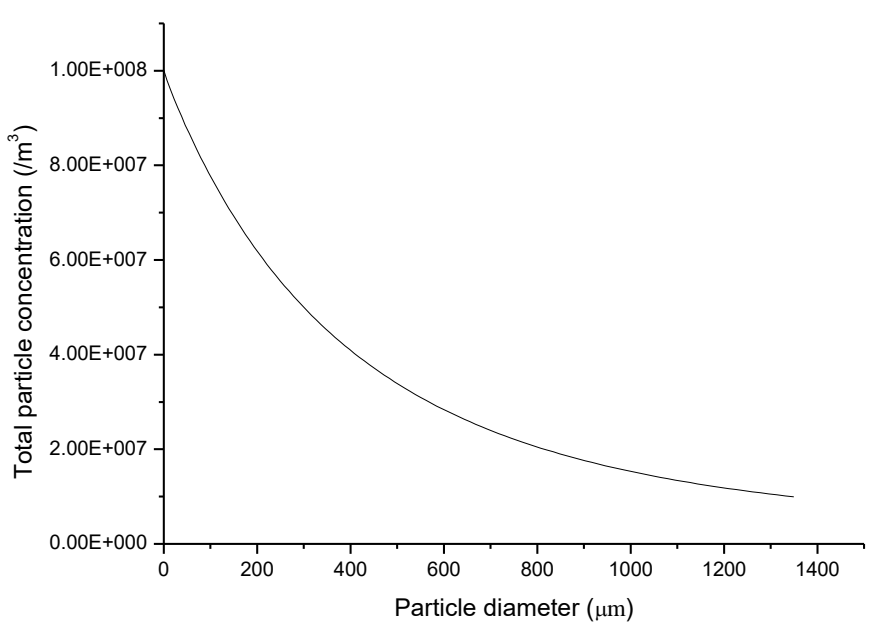

(b)

Fig. 5. (a) Variation of acoustic condensation of natural fog. (b) Variation of total particle concentration. 


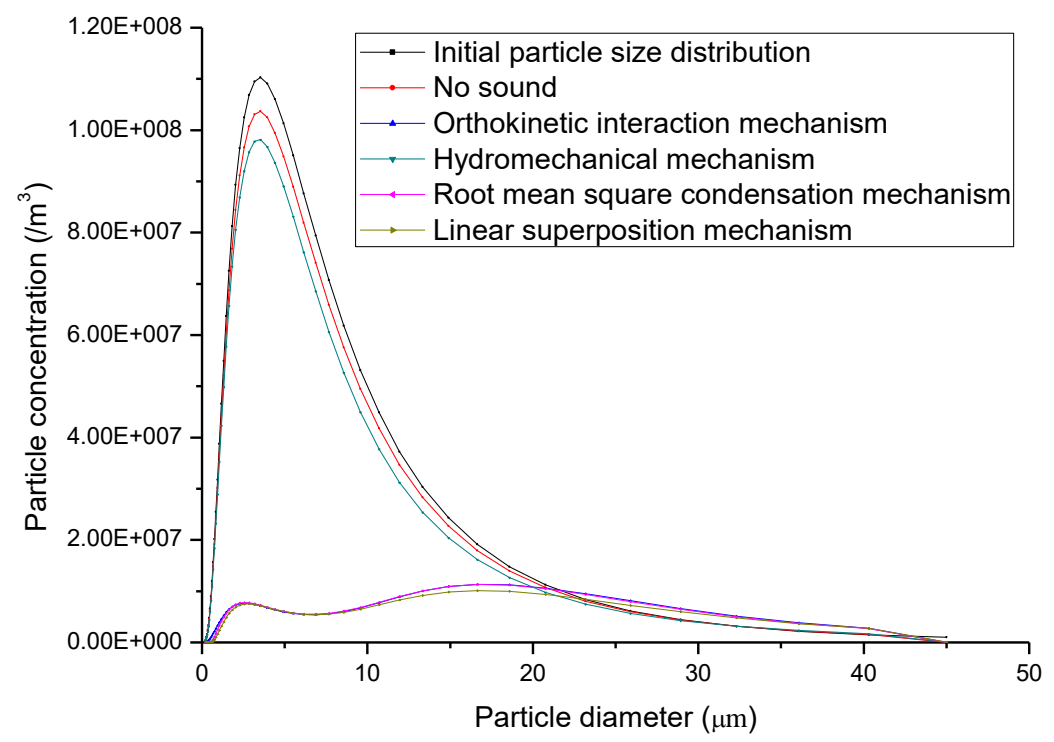

Fig. 6. Condensation effects of different mechanisms.

the most important complementary mechanism to the orthokinetic interaction mechanism, it has a poor effect when considered separately, which confirms the dominant position of the orthokinetic interaction mechanism in the acoustic condensation.

\subsection{Effect of Acoustic Frequency}

The influence of sound frequency on condensation was studied by the control variable method, among which the invariants were: temperature was $20^{\circ} \mathrm{C}$, average diameter of fog drops was $3.5 \mu \mathrm{m}$, minimum particle diameter was $0.2 \mu \mathrm{m}$, maximum diameter was $45 \mu \mathrm{m}$, initial concentration was $100 \mathrm{~cm}^{-3}$, collision efficiency was 1 , number of group was 50 , and sound pressure level was $140 \mathrm{~dB}, 150 \mathrm{~dB}$ and $160 \mathrm{~dB}$ respectively.

Figs. 7, 8, and 9 respectively show the influence of sound frequency on sound condensation after sound waves at different sound pressure levels are applied for the same time. By comparing the condensation effect of different sound pressure levels, it can be found that the particle size distribution diagrams corresponding to different sound pressure levels have similar shapes. Take $160 \mathrm{~dB}$ as an example. Under the action of sound wave, the fog drop particles change from the unimodal distribution at the beginning to the bimodal distribution, reaching the crest at 2-4 $\mu \mathrm{m}$ and 10-16 $\mu \mathrm{m}$, and get to the trough at 4-10 $\mu \mathrm{m}$. High-frequency sound waves are better for condensation of small particles below $6 \mu \mathrm{m}$ and low frequency sound waves are better for the condensation of large particles above $6 \mu \mathrm{m}$, this kind of phenomenon is in conformity with the acoustic condensation mechanism, low frequency acoustic entrainment coefficient is bigger, the ability to trap fog droplets with sound waves is stronger, so for large particles, low frequency's condensation effect is more obvious. High frequency make more collision between particles in unit time and condensation effect is better.

As can be seen in Figs. 7(b), 8(b), 9(b), and 5(b), the acoustic condensation effect causes many small particles to condense into large particles, thus making the number of particles $>20 \mu \mathrm{m}$ is rising, but the overall number is still falling.

According to the theoretical derivation of sound wave condensation (Mednikov, 1965), the effect of frequency on the acoustic condensation efficiency is not a simple linear relationship, but there is an optimal frequency that makes the condensation effect best. Because natural fog concentration is low, and is bimodal size distribution after condensation, the optimal frequency in the picture is not obvious, but through the time that fog condensation effect reached $90 \%$, such as Table 2, we can find the optimal frequency obviously, in the condition of $120 \mathrm{~dB}, 140 \mathrm{~dB}$, $150 \mathrm{~dB}$ and $160 \mathrm{~dB}$, acoustic condensation time gets minimum value at $600 \mathrm{~Hz}$, where $160 \mathrm{~dB}$ is the shortest, 21 minutes can complete condensation, so under the condition of these simulation parameters, acoustic condensation optimal frequency is $600 \mathrm{~Hz}$. 


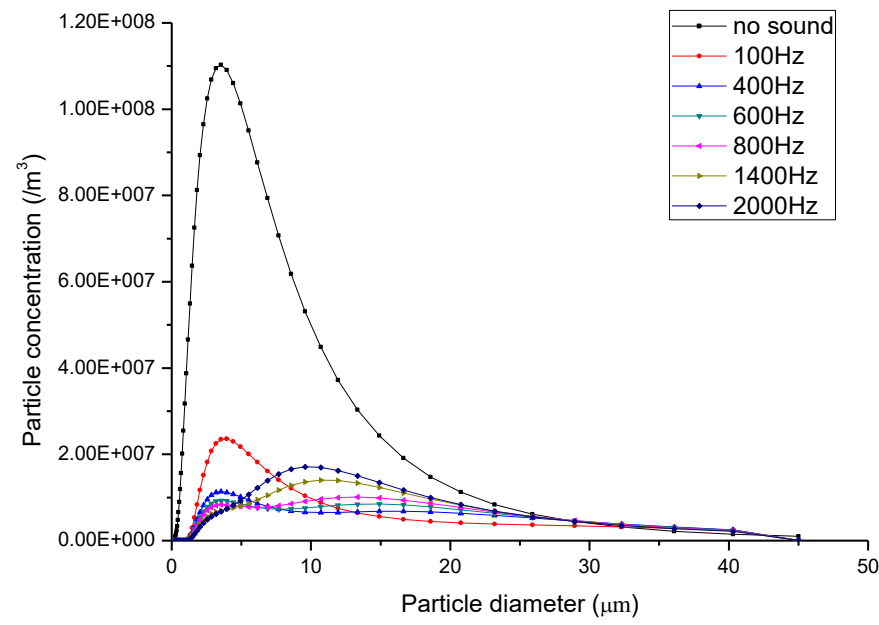

(a)

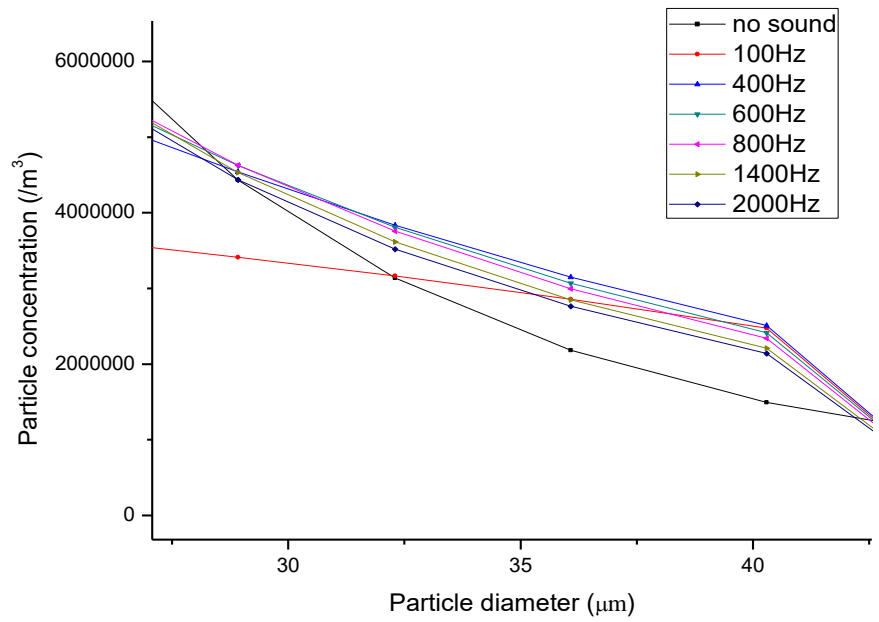

(b)

Fig. 7. (a) $\mathrm{SPL}=140 \mathrm{~dB}$, action time $9500 \mathrm{~s}$, influence of sound frequency on fog droplet size distribution. (b) Local enlarged figure.

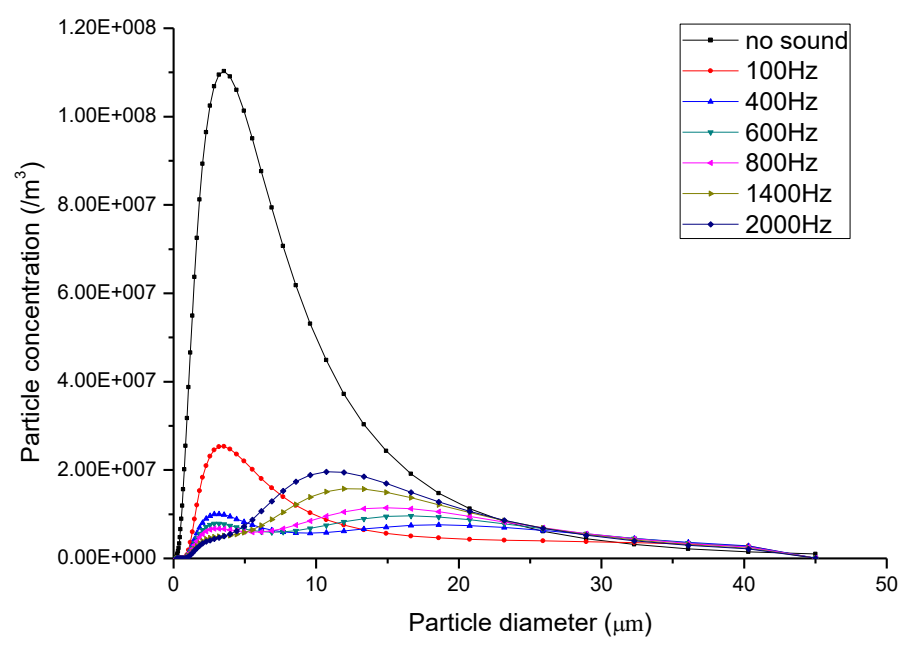

(a)

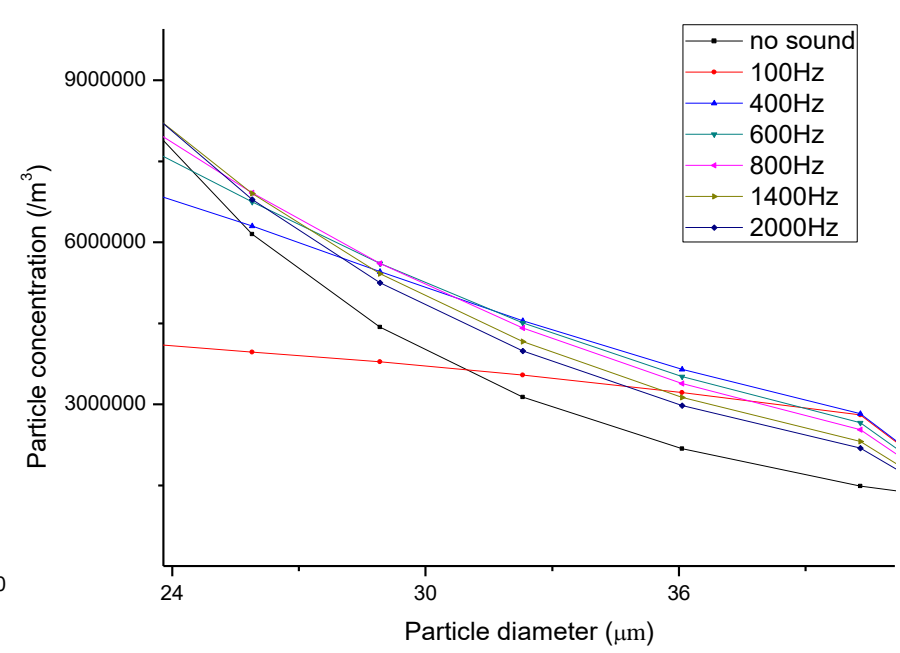

(b)

Fig. 8. (a) SPL = $150 \mathrm{~dB}$, action time $3600 \mathrm{~s}$, influence of sound frequency on fog droplet size distribution. (b) Local enlarged figure.

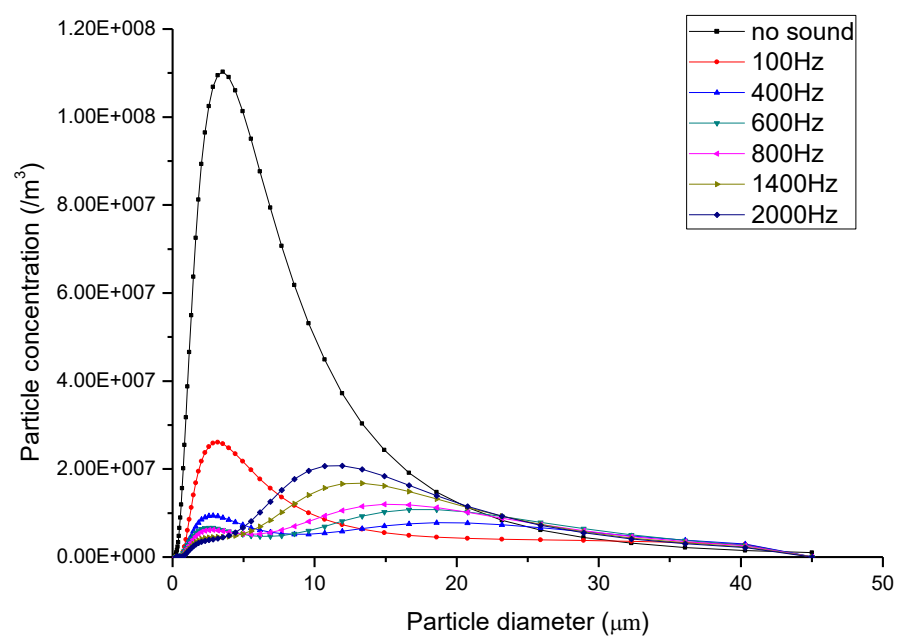

(a)

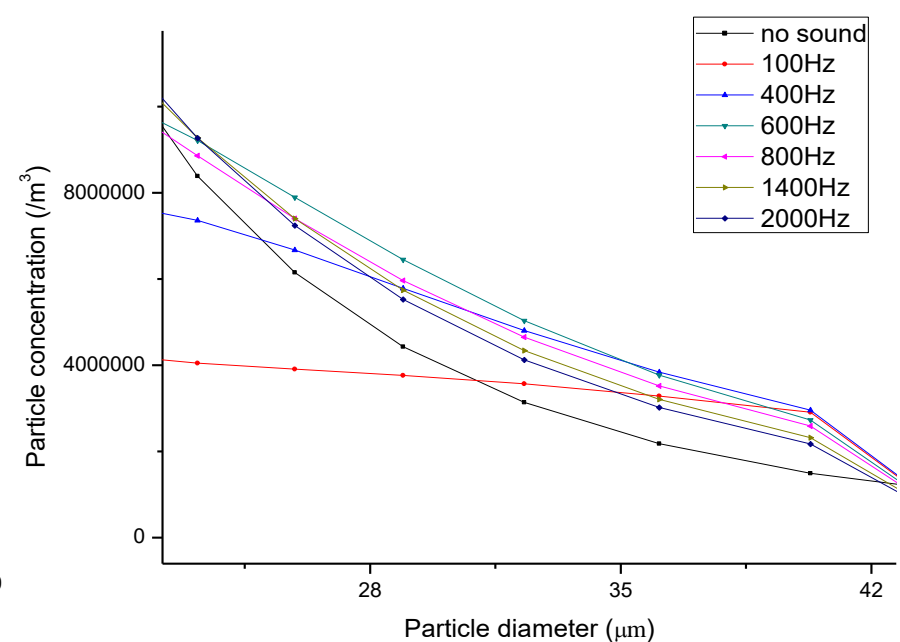

(b)

Fig. 9. (a) $\mathrm{SPL}=160 \mathrm{~dB}$, action time $1300 \mathrm{~s}$, influence of sound frequency on fog droplet size distribution. (b) local enlarged figure. 
Table 2. The time taken for the condensation effect to reach $90 \%$.

\begin{tabular}{lllll}
\hline Time (s) & $160 \mathrm{~dB}$ & $150 \mathrm{~dB}$ & $140 \mathrm{~dB}$ & $120 \mathrm{~dB}$ \\
\hline $100 \mathrm{~Hz}$ & 2304 & 6137 & 14556 & 39613 \\
$400 \mathrm{~Hz}$ & 1326 & 3807 & 9917 & 34889 \\
$600 \mathrm{~Hz}$ & 1280 & 3698 & 9680 & 34553 \\
$800 \mathrm{~Hz}$ & 1292 & 3738 & 9762 & 34624 \\
$1400 \mathrm{~Hz}$ & 1439 & 4146 & 10585 & 35501 \\
$2000 \mathrm{~Hz}$ & 1655 & 4708 & 11647 & 36551 \\
\hline
\end{tabular}

\subsection{Effect of Sound Pressure Level}

The influence of sound pressure level on condensation was studied by the control variable method, among which the invariants were: temperature was $20^{\circ} \mathrm{C}$, average diameter of fog drops was $3.5 \mu \mathrm{m}$, minimum particle size was $0.2 \mu \mathrm{m}$, maximum diameter was $45 \mu \mathrm{m}$, initial concentration was $100 \mathrm{~cm}^{-3}$, collision efficiency was 1 , number of group was 50 , action time was $1000 \mathrm{~s}$, frequency was $100 \mathrm{~Hz}, 600 \mathrm{~Hz}$, and $1400 \mathrm{~Hz}$ respectively.

Comparing Figs. 10, 11, and 12, it can been seen that under the optimal frequency, condensation effect is better at each sound pressure level. Besides, under the condition of $600 \mathrm{~Hz}$ and $1600 \mathrm{~Hz}$, sound wave with $160 \mathrm{~dB}$ has showed a trend of bimodal particle size distribution, condensation effect of sound wave with $1600 \mathrm{~Hz}$ is better than $600 \mathrm{~Hz}$ for particles under $5 \mu \mathrm{m}$, on the other hand, for particles bigger than $5 \mu \mathrm{m}, 600 \mathrm{~Hz}$ is better, this also in line with the conclusion that the sensitivity of different particle sizes to different acoustic frequencies is different.

There are two reasons for the positive correlation between sound pressure level and condensation efficiency: First, with the increase of sound pressure level, the particle soundinduced vibration velocity increases, the particle amplitude increases, the effective volume of particles acting in a sound wave period increases, and the collision efficiency with other particles increases, thus improving the condensation efficiency. Secondly, with the increase of sound pressure level, the relative velocity of periodic vibration between different particles also increases, the collision frequency of particles increases, so the condensation efficiency increases.

\subsection{Effect of Initial Concentration}

The influence of initial concentration on condensation was studied by the control variable method, among which the invariants were: temperature was $20^{\circ} \mathrm{C}$, droplets average diameter was $3.5 \mu \mathrm{m}$, the minimum diameter was $0.2 \mu \mathrm{m}$, the maximum diameter was $45 \mu \mathrm{m}$, collision efficiency was 1 , number of group was 50 , frequency and acoustic pressure level use the optimal

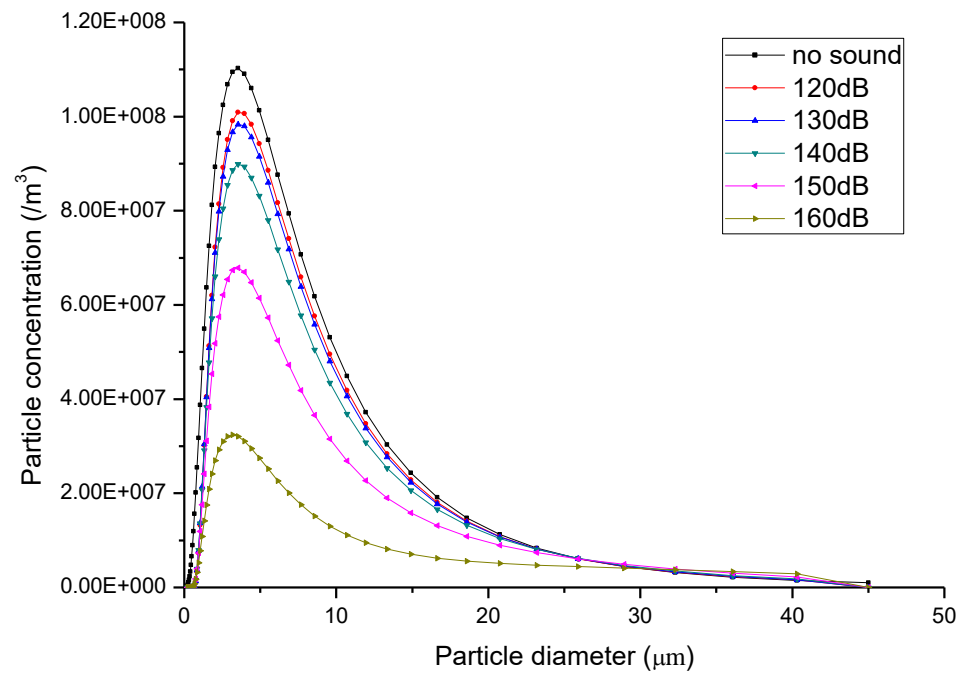

Fig. 10. $f=100 \mathrm{~Hz}$, action time $1000 \mathrm{~s}$, influence of sound pressure level on fog droplet size distribution. 


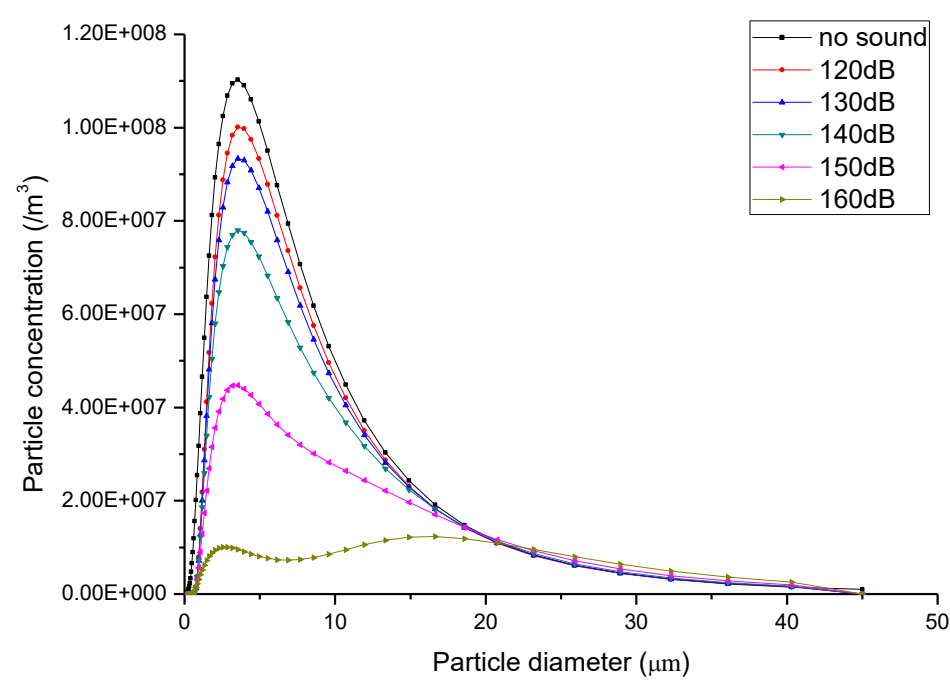

Fig. 11. $f=600 \mathrm{~Hz}$, action time $1000 \mathrm{~s}$, influence of sound pressure level on fog droplet size distribution.

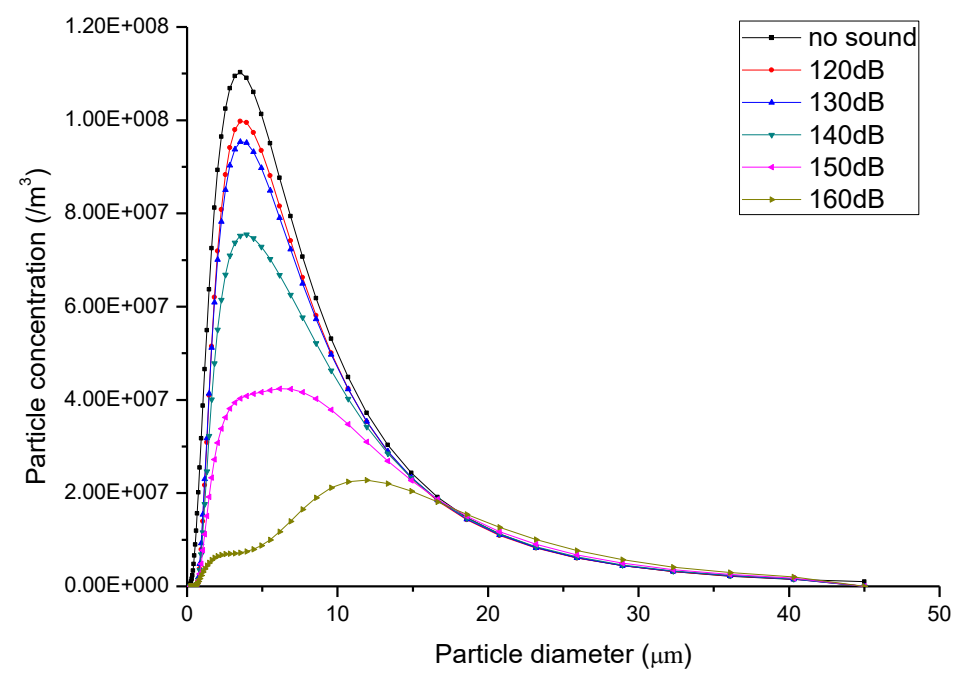

Fig. 12. $F=1600 \mathrm{~Hz}$, action time $1000 \mathrm{~s}$, influence of sound pressure level on fog droplet size distribution.

parameters of $600 \mathrm{~Hz}, 160 \mathrm{~dB}$, which was found in previous two chapters, the action time was $100 \mathrm{~s}, 350 \mathrm{~s}$ and $600 \mathrm{~s}$ respectively, initial concentration use concentration of advection radiation fog $\left(200 \mathrm{~cm}^{-3}\right)$, advection fog $\left(100 \mathrm{~cm}^{-3}\right)$, radiation fog $\left(78 \mathrm{~cm}^{-3}\right)$.

By comparing Figs. 13, 14, and 15, it can be seen that the higher the initial concentration is, the higher the condensation efficiency will be at the same action time. Taking acoustic wave action of $600 \mathrm{~s}$ as an example, the condensation efficiency with the initial concentration of $200 \mathrm{~cm}^{-3}$ is $87 \%$, that with the initial concentration of $100 \mathrm{~cm}^{-3}$ is $74 \%$, and that at the initial concentration of $78 \mathrm{~cm}^{-3}$ is $64 \%$, so the initial concentration is positively correlated with the condensation efficiency. Fog with the initial concentration of $200 \mathrm{~cm}^{-3}$ only takes 10 minutes for the condensation effect to reach $90 \%$, which is 11 minutes shorter than that of $100 \mathrm{~cm}^{-3} \mathrm{fog}$.

As shown in Fig. 13, after the same time interval of $250 \mathrm{~s}$, the condensation efficiency of $100 \mathrm{~s}-$ $350 \mathrm{~s}$ is significantly higher than that of $350 \mathrm{~s}-600 \mathrm{~s}$, which is another manifestation of the positive correlation between the initial concentration and the condensation efficiency. As the concentration of fog drops decreases continuously, the condensation efficiency also decreases. And during condensation process, the particle size distribution has a critical size (Manoucheri and Ezekoye, 1996; Liu et al., 2009) between 20-25 $\mu \mathrm{m}$, below the critical size, particles 


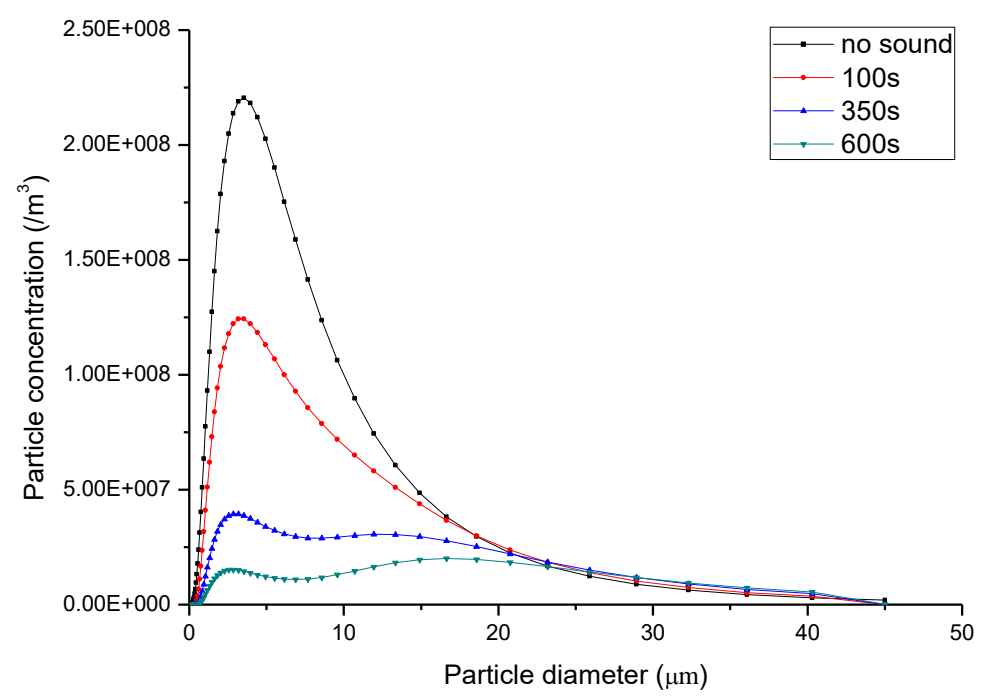

Fig. 13. Initial concentration $=200 \mathrm{~cm}^{-3}$ change of particle size distribution at different moments.

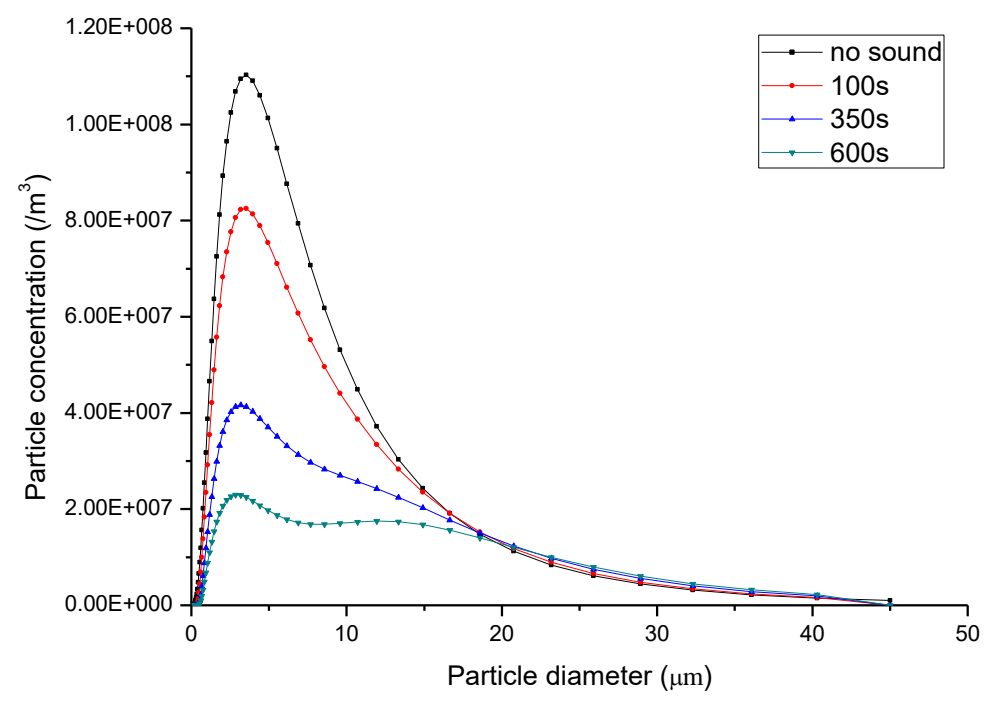

Fig. 14. Initial concentration $=100 \mathrm{~cm}^{-3}$ change of particle size distribution at different moments.

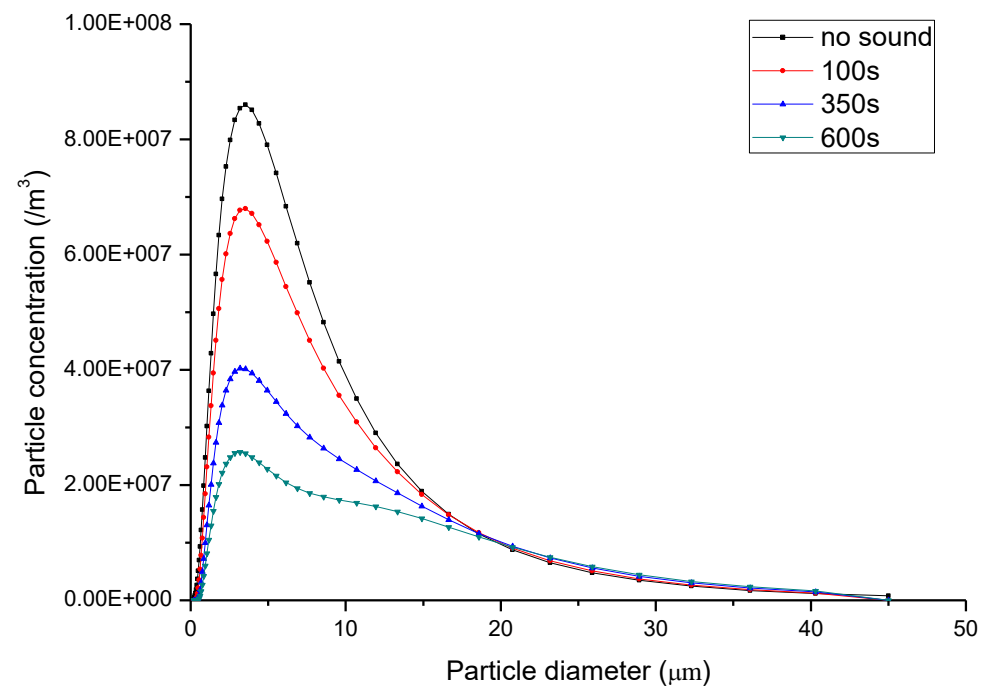

Fig. 15. Initial concentration $=78 \mathrm{~cm}^{-3}$ change of particle size distribution at different moments. 
concentration decreases with sound effect, above critical size, particle concentration inecreases with sound effect, this is because small particles condense into larger particles, largre particles settle, finally achieve the effect of fog dissipation. Speed of condensation of small particles into larger particles is faster than sedimention speed of large particles. From the overall (Fig. 5. (b)), the number of large particles is rising, the total concentration is still falling.

\section{CONCLUSIONS}

This paper proposes the IRMS condensation kernel function, considers orthokinetic interaction mechanism, acoustic wake effect mechanism, mutual radiation mechanism, gravity sedimentation mechanism and Brownian motion mechanism, and fuses them through the method of the root mean square, so that the simulation performance has been improved and more in line with reality. In the simulation process, the measured data of natural fog is selected as the research object, which makes up for the lack of simulation data of natural fog condensation. Through the control variable method, the effects of the sound wave frequency, sound pressure level and initial droplet concentration on the sound wave condensation are studied. First of all, the effects of frequency on condensation efficiency is not linear, there is a optimal frequency $600 \mathrm{~Hz}$, and particle sizes has different sensitivity to different frequency, high frequency has good effect on small particles, and low frequency has good effect on large particles. The particle size distribution diagram is bimodal after the sound wave; Secondly, the sound pressure level is positively correlated with the efficiency of condensation. The higher the sound pressure level, the better the condensation effect. In view of the high energy consumption and strong vibration characteristics of high sound pressure level sound waves, it may cause a certain influence to the stability of the equipment, the economics of fog dissipation and the safety of human body, so it is not appropriate to choose an excessively high sound pressure level. The simulation range of the sound pressure level in this article is below $160 \mathrm{~dB}$; Finally, the initial concentration of particles and the condensation efficiency are also positively correlated. Under the optimal sound wave parameters $600 \mathrm{~Hz}$ and $160 \mathrm{~dB}$, it takes only 11 minutes for the fog of $200 \mathrm{~cm}^{-3}$ to reach $90 \%$ condensation. Because the frequency has different entrainment coefficients for particles of different particle sizes, there is a critical particle size during the condensation process, the concentration of particles above the critical particle size increases and concentration of particles below the critical particle size decreases.

To sum up, this simulation firstly determines the promotion effect of sound wave on fog dissipation, which provides theoretical support for acoustic wave to replace traditional fog dissipation. The simulation model is optimized to provide reference for future research on fog dissipation. By studying the different parameters, the optimal condensation parameters of natural fog dissipation are obtained, which lays a foundation for the practical application of acoustic fog dissipation technology.

\section{ACKNOWLEDGMENTS}

This work is supported by Chinese "13th Five-Year Plan" the third batch of pre-research special technology projects in the space field (305090304HT01).

\section{REFERENCES}

Capéran, PH., Somers, J., Richter, K. (1995). Acoustic agglomeration of a glycol fog aerosol: Influence of particle concentration and intensity of the sound field at two frequencies. J. Aerosol Sci. 26, 595-612. https://doi.org/10.1016/0021-8502(94)00140-T

Cheng, M.T. (1981). Sonic agglomeration of ammonium chloride aerosols in intense sound fields, State University of New York at Buffalo, Buffalo.

Chou, K.H., Lee, P.S., Shaw, D.T. (1981). Aerosol agglomeration and deposition in high-intensity acoustic field. J. Colloid Interface Sci. 83, 335-353. https://doi.org/10.1016/0021-9797(81)903 29-5 
Cleckler, J., Elghobashi, S., Liu, F. (2012). On the motion of inertial particles by sound waves. Phys. Fluids. 24, 033301. https://doi.org/10.1063/1.3696243

Ezekoye, O.A., Wibowo, Y.W. (1999). Simulation of acoustic agglomeration processes using a sectional algorithm. J. Aerosol Sci. 30, 1117-1138. https://doi.org/10.1016/S0021-8502(98)00 778-2

Fan, F., Yang, X., Kim, C.N. (2013). Direct simulation of inhalable particle motion and collision in a standing wave field. J. Mech. Sci. Technol. 27, 1707-1712. https://doi.org/10.1007/s12206013-0420-4

Tandon, P., Rosner D. (1999). Monte Carlo simulation of particle. Aggregation and simultaneous restructuring. J. Colloid Interface Sci. 213, 273-286. https://doi.org/10.1006/jcis.1998.6036

Gao, J.Q., Feng, Y.J., Xiao, W.S. (2010). Techniques and methods of artificial fog elimination. Guangdong Meteorol. 32, 32-34. CNKI:SUN:GDCX.0.2010-01-012

Gelbard, F., Seinfeld, J.H. (1978). Numerical solution of the dynamic equation for particulate system. J. Comput. Phys. 28, 357-375. https://doi.org/10.1016/0021-9991(78)90058-X

González, I., Elvira, L., Hoffmann, T.L., Gallego, J.A. (2001). Numerical study of the hydrodynamic interaction between aerosol particles due to the acoustic wake effect. Acta Acust. 87, 454-460. https://doi.org/10.1134/1.1385426

González, I., Gallego, J.A., Riera, E. (2003). The influence of entrainment on acoustically induced interactions between aerosol particles-An experimental study. J. Aerosol Sci. 34, 1611-1631. https://doi.org/10.1016/S0021-8502(03)00190-3

González, I., Hoffmann, T.L., Gallego, J.A. (2000). Precise measurements of particle entrainment in a standing-wave acoustic field between 20 and $3500 \mathrm{~Hz}$. J. Aerosol Sci. 31, 1461-1468. https://doi.org/10.1016/S0021-8502(00)00046-X

González, I., Hoffmann, T.L., Gallego, J.A. (2002). Visualization of hydrodynamic particle interactions: Validation of a numerical model. Acta Acust. united Acust. 88, 19-26. https://doi.org/10.1134/1.1435561

Hoffmann, T.L. (1997). An extended kernel for acoustic agglomeration simulation based on the acoustic wake effect. J. Aerosol Sci. 28, 919-936. https://doi.org/10.1016/S0021-8502(96)004 89-2

Hoffmann, T.L. (2000). Environmental implications of acoustic aerosol agglomeration. Ultrasonics 38, 353-357. https://doi.org/10.1016/S0041-624X(99)00184-5

Hoffmann, T.L., Koopmann, G.H. (1994) A new technique for visualization of acoustic particle agglomeration. Rev. Sci. Instrum. 65, 1527-1536. https://doi.org/10.1063/1.1144887

Hoffmann, T.L., Koopmann, G.H. (1996). Visualization of acoustic particle interaction and agglomeration: Theory and experiments. J. Acoust. Soc. Am. 99, 2130-2141. https://doi.org/1 $0.1121 / 1.415400$

Hou, S.Q., Wu, J., Xi, B.S. (2002). Experimental study on dissipation of water Mist by lowfrequency acoustic wave. Fluid mech. Exp. Meas. 12, 4. https://doi.org/10.3969/j.issn.16729897.2002.04.010

John, M.W., Peter, V.H. (2006). Atmospheric science (2nd Edition), Academic Press, New York.

Li, X.B., Guan, C.W. (2009). Droplet spectral distribution and infrared attenuation of fog. Laser Infrared. 39, 742-745. https://doi.org/10.3969/j.issn.1001-5078.2009.07.013

Li, Z.H., Niu, S.J. (2001). Research on fog in China in the last 40 years. Acta Meteorol. Sinica 59, 616-623. CNKI:SUN:QXXB.0.2001-05-011

Liu, J., Zhang, G., Zhou, J., Wang, J., Zhao, W., Cen, K. (2009). Experimental study of acoustic agglomeration of coal-fired fly ash particles at low frequencies. Powder Technol. 193, 20-25. https://doi.org/10.1016/j.powtec.2009.02.002

Manoucheri, M., Ezekoye, O.A. (1996). Polystyrene soot agglomeration enhancement in an ultrasonic acoustic field. Hazard. Waste Hazard. Mater. 13, 121-130. https://doi.org/10.1089/ hwm.1996.13.121

Mednikov (1965). Acoustic coagulation and precipitation of aerosols, Consults Bureau, pp. 4-102. https://doi.org/10.4028/www.scientific.net/AMM.687-691.4303

Moore, C.B., Vonnegut, B., Vrablik, E.A., Mccaig, D.A. (1964). Gushes of rain and hail after lightning. J. Atmos. Sci. 21, 646-665. https://doi.org/10.1175/1520-0469(1964)0212.0.CO;2

Niu, S.J., Lu, C.S. (2016). Recent advances in fog research in China. Prog. Meteorol. Sci. Technol. 1, 7-19. https://doi.org/10.3969/j.issn.2095-1973.2016.02.001 
Patel, S.N. (1981). Low intensity $(<155 \mathrm{~dB})$ acoustic agglomeration bench scale experiments, University of New York at Buffalo, Buffalo.

Patterson, H., Cawood, W. (1931). Phenomena in a sounding tube. Nature 124, 667-667. https://doi.org/10.1038/127667a0

Peng, L. (2013). Study on the mechanism of oil mist condensation by ultrasonic wave, Northeast University, Liaoning.

Rajendran, N., Wegrzyn, J., Cheng, M.T., Shaw, D.T. (1979). Acoustic precipitation of aerosol under standing-wave condition. J. Aerosol Sci. 10, 329-338. https://doi.org/10.1016/00218502(79)90048-X

Sahinoglu, E., Tuncay, U. (2013). Increasing coal quality by oil agglomeration after ultrasonic treatment. Fuel Process Technol. 116, 332-338. https://doi.org/10.1016/j.fuproc.2013.07.016

Scott, W. (1968). Analytics studies of cloud droplet coalescence. J. Atmos. Sci. 25, 54-65. https://doi.org/10.1175/1520-0469(1968)0252.0.CO;2

Shaw, D.T., Tu, K.W. (1979). Particle agglomeration due to hydrodynamic interaction between monodisperse aerosols. J. Aerosol Sci. 10, 317-328. https://doi.org/10.1016/00218502(79)90047-8

Sheng, C., Shen, X. (2006). Modelling of acoustic agglomeration processes using the direct simulation Monte Carlo method. J. Aerosol Sci. 37, 16-36. https://doi.org/10.1016/j.jaerosci.2 005.03.004

Sheng, C., Shen, X. (2007). Simulation of acoustic agglomeration processes of poly-disperse solid particles. Aerosol Sci. Technol. 41, 1-13. https://doi.org/10.1080/02786820601009704

Song, L. (1990). Modeling of acoustic agglomeration of fine particles, The Pennsylvania State University, Pennsylvania.

Tamara, T., Svetlana, A. (2015). Acoustical method and device for precipitation enhancement inside natural clouds. Sci. Discovery 3, 18-25. https://doi.org/10.11648/j.sd.s.2015030201.13

Temkin, S. (1994). Gasdynamic agglomeration of aerosols. Phys. Fluids 6, 2294-2303. https://doi.org/10.1063/1.868180

Volk, M. (1977). Sonic agglomeration of aerosol particles, The Pennsylvania State University, Pennsylvania.

Wang, C.B., Li, Q., Chen, H.W. (2007). Numerical simulation and experimental study on the effect of horizontal acoustic wave on $\mathrm{PM}_{2.5}$ particles in gravitational field. Proc. CSEE 27, 18-23. https://doi.org/10.3321/j.issn:0258-8013.2007.23.004

Wang, J. (2012). Study on acoustic agglomeration and other methods to remove fine particles of fly ash from coal burning, Zhejiang University, Hangzhou.

Wu, X.L. (2014). Theoretical and experimental study on acoustic agglomeration of suspended fine particles, National University of Defense Technology, Changsha.

Yao, Y., Su, J.W., Luo, Z.H. (2015) CFD-PBM modeling polydisperse polymerization FBR with simultaneous particle growth and aggregation: The effect of the method of moments. Powder Technol. 272, 142-152. https://doi.org/10.1016/j. powtec.2014.11.037

$\mathrm{Yu}$, J.Z. (2018). Simulation and experimental study on ultrasonic condensation process of water mist, Beijing Jiaotong University, Beijing.

Zhang, G., Liu, J., Wang, J., Zhou, J., Cen, K. (2011). Numerical simulation of acoustic agglomeration by quadrature method of moments. CIESC J. 62, 922-927. https://doi.org/10.1086/433358

Zhang, G., Liu, J., Wang, J., Zhou, J., Cen, K. (2012). Numerical simulation of acoustic agglomeration by improved sectional algorithm. J. Combust. Sci. Technol. 18, 44-49. CNKI:SUN:RSKX.0.2012-01-007

Zhang, G.X. (2010). Theoretical and experimental study on acoustic agglomeration of airborne ash from coal burning, Zhejiang University, Zhejiang.

Zhang, X.R., Gan, C.M., Wei, R.J. (1963). Preliminary experimental study on the effect of acoustic wave on water mist dissipation. J. Nanjing Univ. 3, 21-27 CNKI:SUN:NJDZ.0.1963-05-003

Zheng, J.X., Wang, Z.Q. (2019). Numerical simulation of acoustic agglomeration process of fine particles of coal burning. J. Northeast Dianli Univ. 39, 65-71. CNKI:SUN:DBDL.0.2019-05-010 